\title{
Climate Variability, Dengue Vector Abundance and Dengue Fever Cases in Dhaka, Bangladesh: A Time-Series Study
}

\author{
Sabrina Islam ${ }^{1}$, C. Emdad Haque ${ }^{2, *}$, Shakhawat Hossain ${ }^{3}$ and John Hanesiak ${ }^{4}$ \\ 1 School of Health and Life Sciences, North South University, Dhaka 1229, Bangladesh; \\ sabrina.islam@umanitoba.ca \\ 2 Natural Resources Institute, University of Manitoba, Winnipeg, MB R3T 2N2, Canada \\ 3 Department of Mathematics and Statistics, University of Winnipeg, Winnipeg, MB R3B 2E9, Canada; \\ sh.hossain@uwinnipeg.ca \\ 4 Department of Environment and Geography, University of Manitoba, Winnipeg, MB R3T 2N2, Canada; \\ john.hanesiak@umanitoba.ca \\ * Correspondence: cemdad.haque@umanitoba.ca
}

check for

updates

Citation: Islam, S.; Haque, C.E.; Hossain, S.; Hanesiak, J. Climate Variability, Dengue Vector Abundance and Dengue Fever Cases in Dhaka, Bangladesh: A Time-Series Study. Atmosphere 2021, 12, 905. https://doi.org/10.3390/ atmos12070905

Academic Editors: Jong-Suk Kim, Nirajan Dhakal, Changhyun Jun and Taesam Lee

Received: 15 June 2021

Accepted: 12 July 2021

Published: 14 July 2021

Publisher's Note: MDPI stays neutral with regard to jurisdictional claims in published maps and institutional affiliations.

Copyright: (c) 2021 by the authors. Licensee MDPI, Basel, Switzerland. This article is an open access article distributed under the terms and conditions of the Creative Commons Attribution (CC BY) license (https:/ / creativecommons.org/licenses/by/ $4.0 /)$.

\begin{abstract}
Numerous studies on climate change and variability have revealed that these phenomena have noticeable influence on the epidemiology of dengue fever, and such relationships are complex due to the role of the vector-the Aedes mosquitoes. By undertaking a step-by-step approach, the present study examined the effects of climatic factors on vector abundance and subsequent effects on dengue cases of Dhaka city, Bangladesh. Here, we first analyzed the time-series of Stegomyia indices for Aedes mosquitoes in relation to temperature, rainfall and relative humidity for 2002-2013, and then in relation to reported dengue cases in Dhaka. These data were analyzed at three sequential stages using the generalized linear model (GLM) and generalized additive model (GAM). Results revealed strong evidence that an increase in Aedes abundance is associated with the rise in temperature, relative humidity, and rainfall during the monsoon months, that turns into subsequent increases in dengue incidence. Further we found that (i) the mean rainfall and the lag mean rainfall were significantly related to Container Index, and (ii) the Breteau Index was significantly related to the mean relative humidity and mean rainfall. The relationships of dengue cases with Stegomyia indices and with the mean relative humidity, and the lag mean rainfall were highly significant. In examining longitudinal (2001-2013) data, we found significant evidence of time lag between mean rainfall and dengue cases.
\end{abstract}

Keywords: climate variability; seasonality; dengue fever; vector; rainfall; Bangladesh

\section{Introduction}

The potential impacts of climate change on the human environment and infectious diseases are significant and alarming. Dengue/Severe Dengue Fever (DF/SDF) is one of the most rapidly growing arboviral diseases in the tropics, for which there is currently no universally accepted cure or vaccine. The rapid spread of both the dengue virus (DENV) and its mosquito vector (mostly Aedes aegypti and Aedes albopictus) in the past four decades poses an enormous risk to public health in tropical regions. The Halstead [1] and Gubler [2] studies suggested that the projected emergence will place around 2.5-3.0 billion people at risk of acute illness every year as tropical diseases spread to new areas, such as Europe and North America [3]. As well, Aedes albopictus plays a noticeable role in dengue transmission in the USA and Europe, whereas, in Asia, Aedes aegypti is more dominant in spreading dengue. Presently, populations of 129 countries worldwide are vulnerable with the risk of dengue infection - caused by both kinds of Aedes mosquitoes-of which, $70 \%$ of the actual disease burden exist in Asia. About 5.2 million dengue cases were recorded by the WHO in 2019 with an annual death count of 4032 people. Apart from dengue, these mosquitoes are also vectors of chikungunya, yellow fever and Zika viruses [4].

While some scholars account for climatic factors in their analysis of arboviral disease epidemiology [5,6], climatic factors and/or climate change are generally considered a 
discrete and separate entity in explaining disease dynamics. In clarifying dengue disease epidemiology, some quarters consider climatic aspects as unrelated factors [7]. Several investigations in Asian and Latin American countries, namely in Vietnam [8], Taiwan [9], and Ecuador [10], have confirmed a positive association between Stegomyia indices and Aedes abundance. We argue that climatic factors should therefore be considered as one of the principal determinants of the epidemiological complex that includes vector ecology, pathogen biology, disease transmission, disease occurrence and prevalence, and disease control, prevention, and cure. Such an improved understanding of emerging infectious diseases, including dengue, would enable us to more comprehensively map the process of disease occurrence and spread. This is especially vital for diseases like dengue for which risk assessment, prevention, and control are the only countermeasures available worldwide.

The relationship between climatic conditions and DF/SDF incidence is complex. A meta-analysis of the literature has revealed that rainfall, temperature and humidity are the most important explanatory variables in the transmission of dengue virus (DENV) through the means of vectors (Aedes aegypti and Aedes albopictus) and human hosts [11-13]. However, the specifics of these interactions vary widely from region to region and remain largely inconclusive in the current literature. In addition, several empirical studies in Asia and Latin America cautioned that the relationship between precipitation and dengue incidence may not be linear, as excess rainfall can negatively impact vector breeding $[14,15]$. Additionally, the Aedes mosquito-especially Aedes aegypti-is a type of vector that breeds in clean water and mostly found in different types and sizes of water containers. The role of artificial water containers, especially in urban areas, is therefore very important in Aedes mosquito breeding and dengue incidence. The artificial water containers, especially in the urban areas, thus play a pivotal role in dengue transmission through mosquito breeding, their life cycle, and by infecting people with DENV [16].

By addressing some of the major gaps in previous studies (elaborated on in the following section), our investigation attempts to make a novel contribution by considering the climate-vector-disease nexus in an integrated manner for understanding dengue transmission dynamics. It attempts to determine the effects of the main climate variables (temperature, relative humidity, rainfall, and seasonality) on dengue vector abundance and dengue disease occurrence in the city of Dhaka, Bangladesh. The specific objectives of the study are (1) to examine the relationship between the main climatic factors (temperature, relative humidity, and rainfall) and dengue vector abundance; (2) to examine the relationship between DF/SDF cases and vector abundance; (3) to examine the relationship between climatic factors and DF/SDF cases; (4) to map the patterns in seasonality and climate anomaly, along with their effects on DF/SDF cases.

This paper starts with an overview of the climate factors, specifically temperature, rainfall, relative humidity and dengue relationships, followed by an analysis of the trend in DF/SDF in Bangladesh and a critical review of the relevant studies in the country. The materials and methods are presented in Section 2, followed by the results in Section 3, and an analytical discussion in Section 4, with brief conclusions in Section 5.

\subsection{Climate Factors and Dengue Relationships: An Overview}

\subsubsection{Temperature and Dengue}

The relationship between DENV infection, DF and SDF incidence and temperature follows a complex, nonlinear trend. Most studies on dengue have been conducted in tropical areas where the annual temperature patterns are similar and disease transmission occurs at an optimal temperature range of $20-35^{\circ} \mathrm{C}$ [17-19].

However, vector breeding and disease transmission are dependent on many other socioeconomic and human behavioral factors. It is also critical to distinguish between outside and ambient temperature when examining dengue vector breeding conditions. For example, in sub-tropical regions, breeding can still occur at sub-optimal outside temperatures (i.e., during winter) if the ambient (indoor) temperature is higher and heating and standing water are available [17]. Conversely, even if the outside temperature is optimal, if 
the ambient temperature in buildings is lower due to air conditioning, the vector may not breed or multiply sufficiently to cause an outbreak. The breeding patterns of Ae. aegyptithe major vector for dengue-may not necessarily correlate with outdoor temperature, rather they may be associated with ambient temperature.

\subsubsection{Rainfall and Dengue}

The relationship between dengue incidence and rainfall is dependent on numerous complex, interlinked factors. The dengue epidemiology literature reveals that dengue outbreaks in most countries coincide with the wet season and increased precipitation in general $[12,13,19]$. In this regard, Kuno [17] noticed a positive association between rainfall and larval density and dengue incidence that has since been documented in many tropical countries. However, this causal pathway cannot be universally generalized, as dengue outbreaks follow different climatic patterns in certain regions. Moreover, excess rainfall can negatively impact vector breeding $[14,15]$ by washing off the vector breeding sites and thus can affect dengue outbreaks.

\subsubsection{Relative Humidity and Dengue}

Despite great interest within the research community in the association between climatic factors and dengue incidence, research on relative humidity as an important climatic factor has been relatively scant. Furthermore, the results of the few studies have also been inconsistent and inconclusive. An Indonesian study revealed that the most important predictive factor for dengue outbreaks in that country was relative humidity, with a 3-4-month lag time [14]. This research revealed that low relative humidity during September and October is usually followed by a dengue outbreak early the following year. It is thus highly probable that if seasonal conditions (average temperature and humidity) are shifted due to climate change, seasonal incidences of dengue would be shifted as well.

\subsubsection{Dengue Studies in Bangladesh}

Bangladesh is situated in the tropical monsoon climate zone. Dhaka-the capital city-and other major urban centers experience a hot, wet and humid tropical climate. Bangladesh has a country-wide monsoon mean temperature of about $29^{\circ} \mathrm{C}$ [20], which falls within the optimal range for both mosquito breeding and dengue transmission [17-19]. The major cities of Bangladesh (a country with a population of about 160 million in an area of 143,000 $\mathrm{km}^{2}$ ) have experienced a major resurgence of dengue since 2000 [21,22].

The first epidemic of SDF occurred in 2000 in the cities of Dhaka, Chittagong and Khulna. During this dengue epidemic, a total of 5551 infections were reported and 93 patients died [23]. Since then, serious concern has been expressed regarding the lack of understanding of the dynamics of dengue transmission and the urgent need for improved disease management. According to Sharmin et al. [24], the 2000 outbreak resulted from a virus strain originating in Thailand, located to the east of the country. They also added that dengue cases have remained underreported in Bangladesh as rural people only visit hospitals in the most severe cases. Rahman et al.'s [25] research indicated some degree of correlation between the DF/SDF outbreak in 2000 and monsoon seasonal conditions as the outbreak started in late June 2000, peaked in September (during the rainy season) and decreased in the dry winter season of the same year.

DF/SDF or similar fever is not a new disease in the country. For example, Hossain et al. [22], after analyzing samples from febrile patients between 1996 and 1997, suggested that dengue transmission was ongoing in the country well before 1996. The Sharmin et al. [24] and Morales et al. [26] studies further noted that dengue could be traced back to 1964 in Bangladesh (then East Pakistan)—much earlier than the major outbreak in 2000.

Mortality rates have decreased significantly since the outbreak of 2000; however, a sizeable population is still infected with DENV every year (Figure 1). The distribution of dengue cases and deaths over the period of 2001-2019 in Dhaka is illustrated in Figure 1. 


\section{Trend of Dengue in Dhaka}

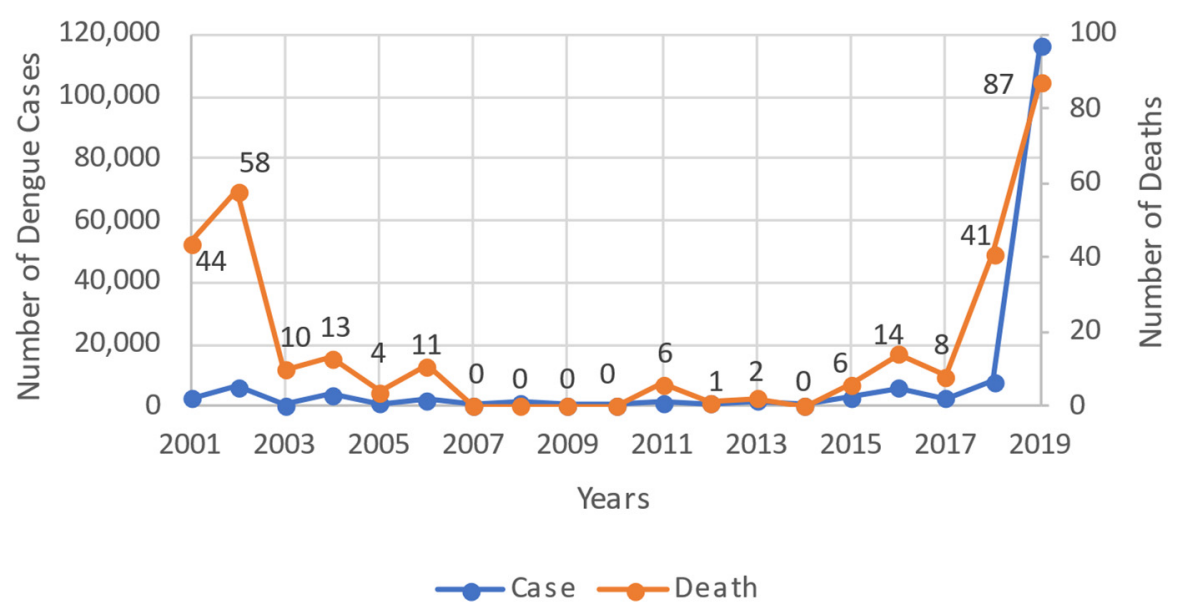

Figure 1. Distribution of dengue cases and number of deaths in the city of Dhaka, Bangladesh, 20012019. (Source: Data procured from the Directorate General of Health Services (DGHS), Bangladesh, 2001-2013; Dhaka Tribune [27]; Shirin et al. [28]).

Due to data unavailability for the year 2000 for Dhaka, both the reported cases and number of fatalities were excluded. Though the dengue incidences have decreased over the years until 2015, it has followed cyclical and fluctuating patterns since 2001. It is evident that the number of deaths has decreased dramatically-from 44 deaths in 2001 and 58 deaths in 2002 to no deaths in 2014. (Figure 1). It then started increasing, resulting in 41 deaths in 2018 and an estimated number of 87 deaths in 2019. There was a large increase in reported dengue cases and number of deaths in 2019. A total of 80,040 dengue cases were reported officially between 1 January and 13 September 2019 with a total of 60 deaths due to SDF and shock syndrome [27]. According to the existing literature, all four DENV serotypes prevail in the city of Dhaka, with DENV-3 being the dominant one [29]. During the 2002 major outbreak, DEN-3 predominated, and subsequently other serotypes were also found to be in circulation.

Studies on the relationship between climate and dengue transmission in Bangladesh have primarily focused on patients in hospitals and clinics [22,24,25,30], and only a few investigations have hitherto been carried out in the country [31-33]. Paul et al. [34] studied the effects of climatic factors on Aedes abundance in the city of Dhaka, Bangladesh, limiting their study only to climate factors and vector abundance relationships. They concluded that rainfall, temperature, and relative humidity significantly affected the mean abundance of mosquitoes.

In a rare study on climate-dengue case relationships in Bangladesh, Hashizume et al. [31] conducted a time-series analysis of the trend between hydro-climatological variability and DF cases, and found a positive association between DF cases with high as well as very low river levels with varying weekly time lags of $0-19$ weeks. Islam et al.'s [35] recent study of the city of Dhaka inferred that dengue incidence is significantly associated with the monthly mean temperature, total rainfall, and mean humidity. The study established a linear relationship of the climatic factors and the dengue incidence, while not accounting for the aspects of seasonality and the vector relationships.

Karim et al. [32] examined the influence of climatic factors on dengue cases in the city of Dhaka, and found that rainfall, maximum temperature, and relative humidity could explain $61 \%$ of the variability in reported dengue cases with a two-month lag period. The study revealed that the arrival of the monsoon season, with a peak in August, was sufficient to explain most of the reported dengue cases. However, the role of vector mosquitoes in dengue transmission was not considered. An investigation in the city of Dhaka revealed 
that both Ae. aegypti and Ae. albopictus larval populations peaked in July at the height of the monsoon [36].

In studies on dengue in Bangladesh, we argue that the focus has generally been on bivariate relationships either between climate factors and vector abundance or between vector abundance and dengue cases [24,32,33]. In Bangladesh studies, the climate-vectordisease nexus under one research framework has not yet been explored. We therefore assert that considerable gaps still exist in our understanding of the complex climate-vectordisease nexus and how these relationships are being affected by confounding factors such as urbanization and human behavior. As there is not yet a cure or universally available vaccine for dengue, it is vital to improve our understanding of risk factors in order to effectively control and prevent the spread of the disease in developing countries like Bangladesh.

\section{Materials and Methods}

\subsection{Study Area and Design}

The city of Dhaka is the largest urban center of Bangladesh. Considering its socioeconomic, political and demographic significance, pivotal standing in terms of population health risk to infectious diseases, and recurring number of dengue cases, the Dhaka City Corporation (DCC) was chosen as the study area for this investigation (Figure 2). Located on the banks of the Buriganga River, Dhaka has an area of 126.34 square kilometers (census 2011) [37] and ranks 11th among global mega-cities with a population of 18.2 million [38]. The city experiences a hot, wet, and humid tropical climate and a distinct monsoon season, with an annual average temperature of $28^{\circ} \mathrm{C}\left(82^{\circ} \mathrm{F}\right)$ and monthly means varying between $20^{\circ} \mathrm{C}\left(68^{\circ} \mathrm{F}\right)$ in January and $32{ }^{\circ} \mathrm{C}\left(90^{\circ} \mathrm{F}\right)$ in May. Nearly $80 \%$ of the annual average rainfall of $1854 \mathrm{~mm}$ (73 in) occurs between May and September [39].

\subsection{Data Collection Techniques}

First, the meteorological data required for the study included temperature, relative humidity and rainfall on daily, monthly and yearly time scales. These data were obtained from the Bangladesh Meteorological Department (BMD) in Dhaka for the 1985-2014 period. A near standard 29-year period (1985-2014) was used as the climate baseline to calculate climate anomalies in relation to dengue cases [40]. We could use only 29 years of data instead of 30 years as data prior to 1985 were not available from the BMD. The data collection method was different before 1985 and therefore data were not compatible with data available from 1985 onwards. The data were obtained from a single observatory located at the Dhaka Airport (Old) (Figure 2). Monthly averages were calculated from the daily data for trend and seasonality analysis of the selected variables (temperature, rainfall and humidity) in relation to dengue cases.

Notably, we could not use daily data since daily data were not available for the Aedes mosquito or dengue cases. The data were homogeneous according to the BMD for the period mentioned. Second, the data for the House Index (HI), Breteau Index (BI) and the Container Index (CI) for Ae. aegypti larvae for the city of Dhaka were obtained from the Directorate General of Health and Services (DGHS) of the Government of Bangladesh for the 2002-2013 period. There were two limitations: (i) continuous time series data were not available as entomological surveys were conducted with interruptions, and (ii) there were common as well as uncommon surveyed areas in the sequential surveys. Therefore, the larval data from entomological surveys were available only for seven years: 2002, 2003, $2004,2005,2009,2012$ and 2013. These surveys were carried out during the same period (monsoon season) of each year, namely during June-October months. 


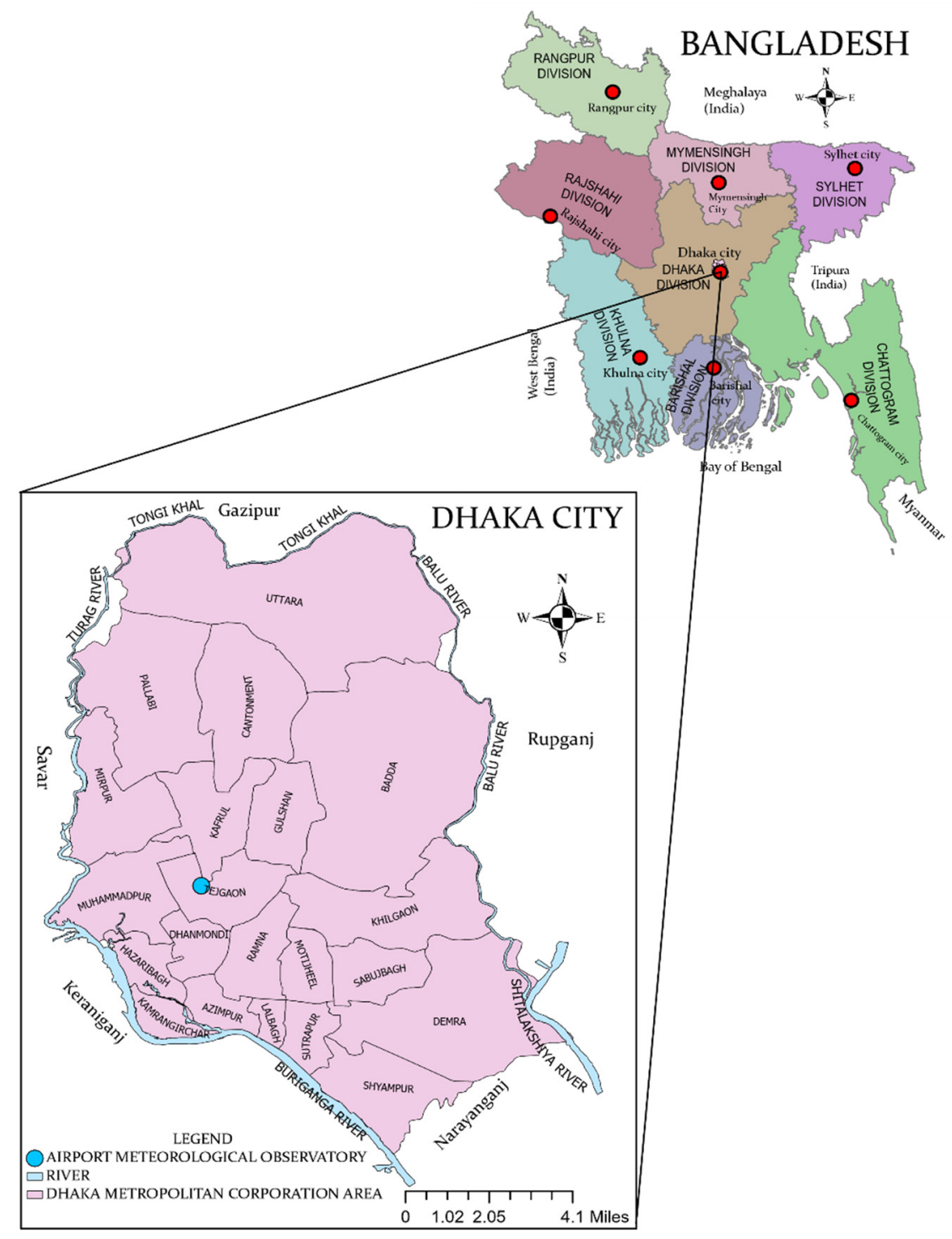

Figure 2. Location map of the study area-the City of Dhaka, Bangladesh.

However, as noted above, the geographic areas surveyed by the DGHS had common as well as varied areas from year to year. In order to maintain consistency, ensure comparability and reduce possible biases, we selected only the common areas within which data collection was repeated for at least five years. We re-calculated all indices (HI, BI and CI) for the city of Dhaka based on DGHS datasets. As no data were available for 2006, 2007, 2008, 2010, and 2011 from any sources in Bangladesh to conduct a field-data based time-series analysis, these missing data were denoted as "missing at random". Following Little and Rubin [41] and Weerasinghe [42], these missing data were imputed by applying the Spline Interpolation Method (see R-package "imputTS") [43,44] and the regression imputation method [45]. Third, the dengue case data (2002-2013) were also obtained from the DGHS. All statistical analyses were performed using Microsoft Excel and the statistical software R [46].

\subsection{Statistical Analyses}

We applied various statistical techniques to identify interactions within the climatevector-disease nexus. To the best of our knowledge, this relationship has not been previously studied to a significant extent, especially in the South Asian context, and thus the constituent dynamics are not understood well. Our initial approach is to analyze the effects of climatic variables on vector abundance, then subsequently analyze the effect of vector 
abundance on dengue incidence, conceptualizing this pathway as a step-by-step process. In addition, we also analyzed seasonality factors that may affect disease patterns over the years. To implement the above, the following data analyses were performed based on the collected meteorological, entomological and dengue case data:

Analysis of climate factors vs. vector indices: first, we calculated the monthly mean temperature (MT), mean relative humidity (MH), mean rainfall (MR), and lag mean rainfall (LMR) of one-month lag. We then attempted to find relationships between each of the indices (CI, BI and $\mathrm{HI}$ ) and the climate factors (MT, MH, MR, and LMR) for the period of 2002-2013. Linear regression model assumes a fixed parametric form of the relationship between vector indices and climate factors. The generalized additive model (GAM) [47] does not assume any specific form of this relationship and can be used to reveal and estimate nonlinear effects of the climate factors on the vector indices. To implement this relation, the following GAM was used:

$$
\text { Index }_{i t}=\beta_{0}+f\left(M_{t}\right)+f\left(M_{t}\right)+f\left(M R_{t}\right)+f\left(L_{M R}\right)+\text { error }_{t}
$$

where $\beta_{0}$ is the intercept; i is $\mathrm{HI}, \mathrm{CI}$ or $\mathrm{BI} ; \mathrm{t}$ is time; $\mathrm{f}$ is the unknown smooth functions of climatic factors which are determined by the data.

We used an autocorrelation of lag 1 to measure the relationship between one month's temperature $\left(Y_{t}\right)$ and the previous month's temperature $\left(Y_{t-1}\right)$ (same for humidity, rainfall and lag mean rainfall). The values of the autocorrelation function (ACF) and partial autocorrelation function (ACF) helped us to identify the autoregressive order and moving average order, respectively. Next, we estimated the GAM model parameters after adjusting the order in the estimation procedure.

Analysis of vector abundance vs. dengue case incidence: here, we emphasized to examine the relationship between vector abundance (by using $\mathrm{HI} / \mathrm{BI} / \mathrm{CI}$ ) and dengue cases for the period of 2002-2013. Since our response (dengue cases) was counts, the following Poisson regression model was used to model vector abundance vs. dengue case incidence:

$$
\log (\mu \mathrm{t})=\beta_{0}+\beta_{1} \mathrm{HI}_{\mathrm{t}}+\beta_{2} \mathrm{BI}_{\mathrm{t}}+\beta_{3} \mathrm{CI}_{\mathrm{t}}
$$

where $\mu t$ is the mean case count with respect to time . $_{\text {. }}$

Analysis of climate factors vs. dengue case incidence: we examined the relationship between dengue cases and climatic factors for specific months over the 12-year study period (2002-2013), using the Poisson regression model. In statistics, when modeling count data (number of dengue cases, in our study), the Poisson regression model is used.

The relationship among the climate-vector-dengue nexus was established based on the above modeling approaches. However, we could not consider seasonality effects in this analysis because appropriate vector data for seasonality were unavailable. For seasonality analysis, we used a different dataset which has monthly average temperature, rainfall and relative humidity data for the period of 2001-2013. A relationship of climatic factors and dengue cases was established after adjusting the seasonality effect.

Analysis of seasonality vs. dengue cases: dengue case data for Dhaka city were plotted against the climate data variables (temperature, rainfall and relative humidity) from the BMD for the period of 2001-2013 in order to map seasonal variations in dengue cases. We then determined the relationship between dengue cases (which is again count response) and climatic factors (MT, MH, and MR), using the following Poisson regression model:

$$
\log (\mu \mathrm{t})=\beta_{0}+\beta_{1} \mathrm{MT}_{\mathrm{t}}+\beta_{2} \mathrm{MH}_{\mathrm{t}}+\beta_{3} \mathrm{MR}_{\mathrm{t}}
$$

where $\mu t$ is the mean case count with respect to time ${ }_{t}$. After establishing the climatevector-dengue nexus for specific months over 2002-2013 and the seasonal variation of dengue while determining the effects of climatic factors on dengue, it is important to examine the variation in dengue cases based on climate anomalies (of annual temperature and rainfall over the period of 2001-2013). To implement this, we calculated the annual 
mean of temperature and rainfall for the period 1985-2013 to act as the climate normal baseline for the study. The annual anomaly was found by subtracting the climate mean of temperature, relative humidity and rainfall from the data for each individual year. This can be expressed as:

$$
\text { annual anomaly }_{(2001-2013)}={\text { annual } \operatorname{mean}_{(2001-2013)}-\operatorname{climate}_{\text {normal }}(1985-2013)}
$$

The data obtained from relation (4) were then plotted to show the annual anomaly over the 2001-2013 period. This depicted the changes in dengue disease occurrence in association with the annual anomaly over the years for the 2001-2013 period.

Notably, prior to a major dengue outbreak in 2000, case data collection by the public health agencies was sporadic and limited to clinical data. Large scale (population-based) dengue case data collection began only after the outbreak in 2000. Subsequent to the 2000 dengue outbreak, large scale entomological data collection was also initiated and were available from 2002 onward. Limited by data unavailability, we used the 13-year period (2001-2013) for examining the relationships between seasonality and dengue cases, and a 12-year period (2002-2013) for examining the relationship between entomological data-based vector abundance with dengue cases, and the climate variables. These time frames are graphically shown in Figure 3 for clarity.

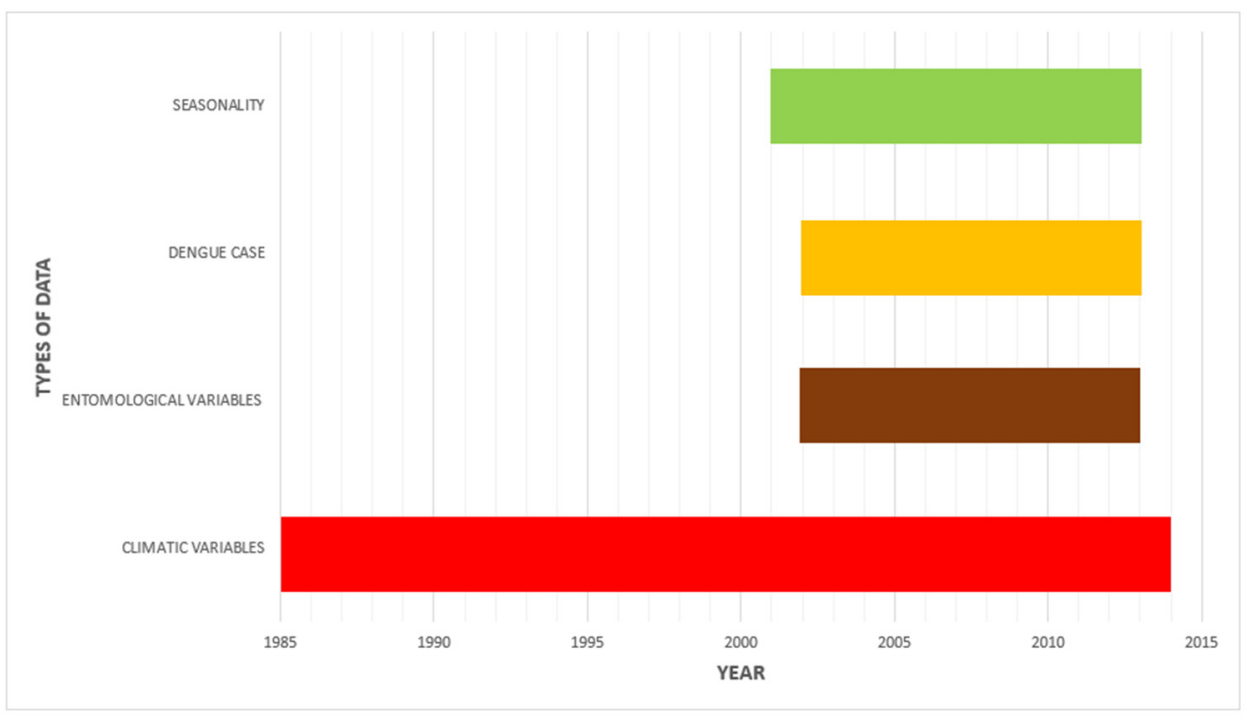

Figure 3. Overlapping time periods of different datasets.

This research was approved by the Bangladesh Medical Research Council (Bangladesh) and the Joint Faculty Research Ethics Board of the University of Manitoba (Canada). Administrative permission was granted by the Government of Bangladesh to access and use the meteorological data from the Bangladesh Meteorological Department, and entomological and dengue case data from the Directorate of Public Health and Services.

\section{Results}

In this section we provide the data analysis results based on the sequence of analyses discussed in the previous section.

\subsection{Analysis of Climate Factors vs. Vector Indices}

We examined the effects of climatic factors on each of the Stegomyia indices (CI, BI and HI) for the city of Dhaka over the 2002-2013 period, based on observed (by BMD and DGHS) and our imputed meteorological and entomological data (Table 1). 
Table 1. Vector indices and climatic variables for specific months (2002-2013) in the city of Dhaka, Bangladesh.

\begin{tabular}{|c|c|c|c|c|c|c|c|c|}
\hline Years & Months & $\% \mathrm{CI}$ & $\%$ BI & $\% \mathrm{HI}$ & MT $\left({ }^{\circ} \mathrm{C}\right)$ & MH (\%) & MR (mm) & LMR (mm) \\
\hline 2002 & Aug. & 7.49 & 15.28 & 14.25 & 28.6 & 81 & 8.77 & 14.39 \\
\hline 2003 & Aug. & 16.31 & 16.84 & 8.74 & 29.4 & 78 & 6.52 & 6.16 \\
\hline 2004 & Jun. & 29.91 & 32.54 & 14.04 & 28.5 & 81 & 15.87 & 5.23 \\
\hline 2005 & Sep. & 15.7 & 13.91 & 10.47 & 28.9 & 81 & 17.13 & 11.65 \\
\hline 2006 & Aug. & 8.11 & 14.69 & 8.49 & 29.1 & 77 & 5.39 & 10.68 \\
\hline 2007 & Aug. & 13.61 & 39.14 & 10.73 & 29.1 & 80 & 16.29 & 24.29 \\
\hline 2008 & Aug. & 26.46 & 71.07 & 15.58 & 28.8 & 81 & 10.29 & 18.16 \\
\hline 2009 & Jun. & 40.91 & 94.29 & 21.43 & 30.2 & 74 & 5.67 & 5.42 \\
\hline 2010 & Aug. & 51.56 & 96.59 & 26.65 & 29.5 & 78 & 10.97 & 5.39 \\
\hline 2011 & Aug. & 54.36 & 81.7 & 29.45 & 28.5 & 82 & 13.19 & 11.48 \\
\hline 2012 & Sep. & 45.6 & 57.33 & 28.01 & 29 & 79 & 2.70 & 9.10 \\
\hline 2013 & Oct. & 21.47 & 30 & 20.53 & 27.2 & 78 & 4.23 & 5.73 \\
\hline
\end{tabular}

Note: The specific months in this study were used because data were available only for these months as collected and provided by the DGHS.

Results of our univariate analysis revealed that there is a nonlinear relationship between CI and mean rainfall (MR) or lag mean rainfall (LMR). We used a smoothing method based on cubic splines to estimate the functional effect of climate factors on the vector indices GAM (1).

The results of the fitted model (1) showed that neither mean temperature (MT) nor mean relative humidity ( $\mathrm{MH}$ ) were significantly associated with $\mathrm{CI}$. However, MR was found to be positively associated with CI (Table 2). The adjusted $\mathrm{R}^{2}=0.79$ means that the fitted model can explain $79 \%$ of the variability in CI due to MR.

Table 2. Association between the Stegomyia indices and climate variables, dengue cases with the Stegomyia indices and dengue cases with climate variables.

\begin{tabular}{|c|c|c|c|c|c|c|c|c|c|c|c|c|}
\hline \multicolumn{13}{|c|}{ Association of Stegomyia Indices with Climate Variables } \\
\hline & \multicolumn{4}{|c|}{ Association with CI } & \multicolumn{4}{|c|}{ Association with BI } & \multicolumn{4}{|c|}{ Association with HI } \\
\hline Variables & MT & $\mathrm{MH}$ & MR & LMR & MT & $\mathrm{MH}$ & MR & LMR & MT & $\mathrm{MH}$ & MR & LMR \\
\hline$p$-value & 0.294 & 0.360 & $0.0372 *$ & 0.0762 & 0.7976 & $0.0208 *$ & 0.0186 * & 0.8572 & 0.448 & 0.259 & 0.197 & 0.678 \\
\hline $\mathrm{R}^{2}$ (adj) & 0.79 & & & & 0.72 & & & & & & & \\
\hline \multicolumn{13}{|c|}{ Association of dengue cases with Stegomyia Indices } \\
\hline Variables & $\mathrm{HI}$ & BI & CI & & & & & & & & & \\
\hline$p$-value & 0.005 * & $<0.001$ * & $<0.001 *$ & & & & & & & & & \\
\hline $\mathrm{R}^{2}$ (adj) & 0.49 & & & & & & & & & & & \\
\hline \multicolumn{13}{|c|}{ Association of dengue cases with climate variables } \\
\hline Variables & LMR & $\mathrm{MH}$ & & & & & & & & & & \\
\hline$p$-value & $\begin{array}{c}0.0279 \\
*\end{array}$ & 0.0463 * & & & & & & & & & & \\
\hline $\mathrm{R}^{2}$ (adj) & 0.93 & & & & & & & & & & & \\
\hline
\end{tabular}

Note: * significant at $0.05 . \mathrm{HI}=$ No. of positive $\mathrm{HHs} /$ no. of $\mathrm{HHs}$ visited, $\mathrm{CI}=$ No. of positive containers $/$ No. of wet containers, $\mathrm{BI}=\mathrm{No}$. of positive containers/No. of HHs visited. $\mathrm{MR}=$ mean rainfall, $\mathrm{MH}=$ mean humidity, $\mathrm{LMR}=$ lag mean rainfall for one month.

To check the normality of the error term in the fitted model (1), we drew the autocorrelation (ACF) and partial autocorrelation plots (PACF) of residuals. The PACF plot (Figure S1) indicated that the residuals in the fitted model were uncorrelated with mean zero and constant variance.

We fitted the same model (1) when the response was BI. Both MT and LMR showed positive relationships with BI but were not significant. However, the results indicated that $\mathrm{BI}$ was significantly related to $\mathrm{MH}$ and MR (Table 2). The MH was negatively related to BI, meaning that as $\mathrm{MH}$ increases, $\mathrm{BI}$-and thus vector density-decreases. The adjusted $\mathrm{R}^{2}$ value $=0.72$ means that the fitted model can explain $72 \%$ of the variability in BI 
associated with $\mathrm{MH}$ (Table 2). We checked the model assumption using ACF and PACF plots, which revealed that the residuals were uncorrelated with mean zero and constant variance (Figure S2).

We fitted the same model (1) again when the response was HI, revealing that there were positive relationships between $\mathrm{HI}$ and MT, MR and LMR, and a negative relationship with MH. None of the climatic factors were significantly related to HI (Table 2). The above findings reveal how dengue vector abundance and distribution are impacted by temperature, rainfall, and relative humidity. As the vector is a means rather than a final phenomenon in terms of dengue disease, it is critical to extend our examination to the relationship between dengue and the Stegomyia indices.

\subsection{Analysis of Vector Abundance vs. Dengue Case Incidence}

We fitted the Poisson regression model (2) of dengue cases with each of the indices HI, $\mathrm{BI}$, and CI. The results indicated that there were highly significant relationships between dengue cases and the indices (HI, BI and CI) (Table 2). It is evident from the results that both BI and CI were highly significant variables among the three indices. The adjusted $\mathrm{R}^{2}$ value of 0.489 showed that the model could explain about $49 \%$ of the variability in dengue cases accounted for by the indices (Table 2). We examined the ACF and PACF plots of standardized Pearson residuals of the fitted model (2), which revealed that the residuals behaved as uncorrelated with mean zero and constant variance.

\subsection{Analysis of Climate Factors vs. Dengue Case Incidence}

We fitted the Poisson regression model (2) of dengue cases with each of the climatic factors MT, MH, MR, and LMR. We present below the results from the estimated model, which reveal the relationships between dengue cases and climatic factors for the study period (i.e., 2002-2013) for specific monsoon months, encompassing June to August.

Results from the fitted model (2) indicate that there are significant relationships between dengue cases and each of MH, and LMR (Table 2). It is evident from these results that $\mathrm{MH}$ and LMR are significantly related to dengue cases. The adjusted $\mathrm{R}^{2}=0.93$ means that the model can explain about $93 \%$ of the variability in dengue cases resulting from climatic factors (Table 2). We examined the ACF and PACF plots of standardized Pearson residuals for the fitted model (2), which indicated that the residuals behaved as uncorrelated with mean zero and constant variance.

\subsection{Analysis of Seasonality vs. Dengue Cases}

After analyzing the relationship among the climate-vector-dengue nexus above based on the available DGHS data only during monsoon season, we considered another dataset; because the previous dataset only had data for monsoon seasons, and for seasonality analysis, we needed monthly data for all the climatic variables for the period considered under the study. This was used to analyze seasonal variability of dengue cases versus monthly average temperature, rainfall and relative humidity for the 2001-2013 period. Here, we analyzed the seasonality in terms of dengue cases only and determined the relationship of climatic factors and dengue cases while taking the seasonality in account.

First, the monthly time series of temperature, relative humidity and rainfall data along with dengue cases were plotted in Figure 4 to view the patterns in their distribution over a 13-year period (2001-2013). Figure 4a indicates that dengue cases follow a peak returning pattern, with a sharp rise (of more than 3000) in the second year of the study (2002) and peak every other year until 2008, after which the peaks occurred every two years. Figure $4 \mathrm{~b}$ reveals that temperature followed a similar pattern over the same 13-year period, with a peak of $30^{\circ} \mathrm{C}$ (monthly average temperature) during months of July / August of most of the years and a low of $16^{\circ} \mathrm{C}$ (monthly average temperature) in early 2003. Figure $4 \mathrm{c}$ shows the monthly average relative humidity pattern over the 13-year study period, which was cyclical with a consistent yearly peak of $80-85 \%$. Finally, Figure 4 d also depicts a yearly cyclical and seasonal pattern of rainfall. The average monthly rainfall for 
the aforementioned period varies between 100 and $150 \mathrm{~mm}$ with occasional departures; for example, the peaks in 2004, 2006, 2007 and 2009 with highest peak in 2004 having more than $250 \mathrm{~mm}$ monthly average rainfall.

(a): Number of dengue cases

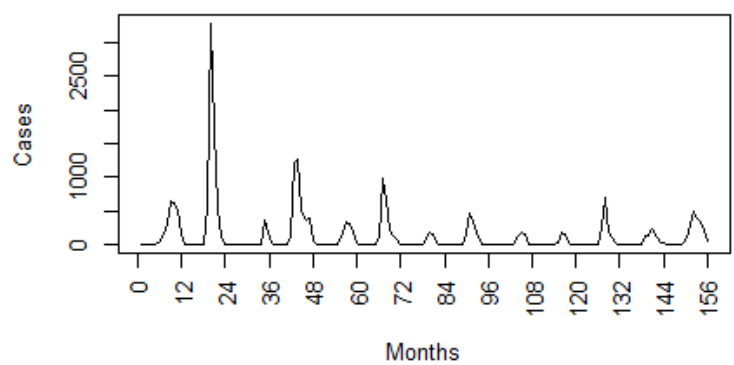

(c): Monthly average humidity

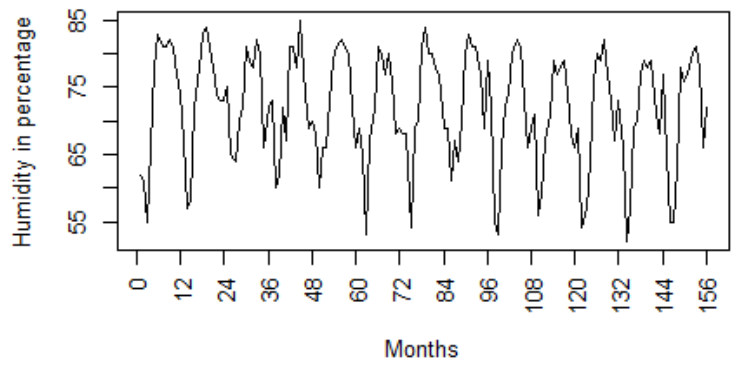

(b): Monthly avearge temperature

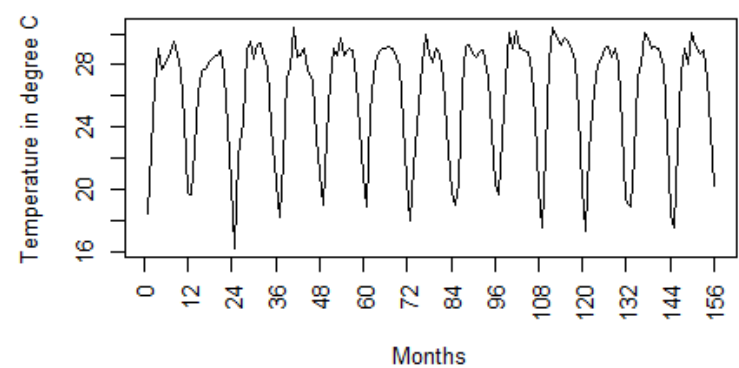

(d): Monthly average rainfall

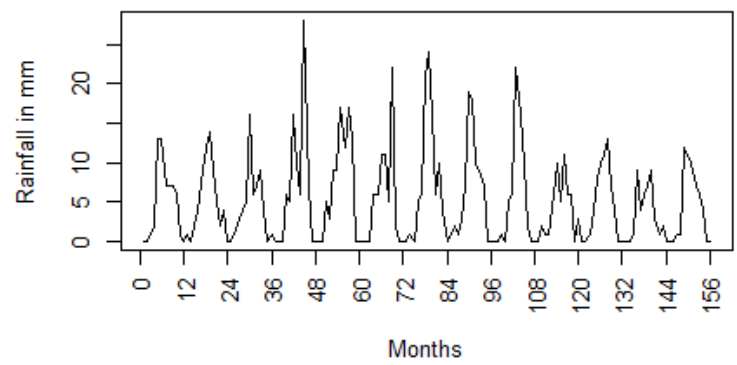

Figure 4. Patterns in the distribution of monthly data for dengue cases and selected climate factors for the 20012013 period in the city of Dhaka, Bangladesh: (a) number of dengue cases; (b) average monthly temperature; (c) average monthly relative humidity; (d) average monthly rainfall. Note: Every 12 months $=1$ year, starting from year 2001 to year 2013. Thus, months 0-12 = year 2001, months 12-24 = year 2002, months 24-36 = year 2003, months 36-48 = year 2004, months 48-60= year 2005, months 60-72 = year 2006, months 72-84= year 2007, months 84-96 = year 2008, months 96-108 = year 2009, months 108-120 = year 2010, months 120-132= year 2011, months 132-144 = year 2012, months 144-156 = year 2013.

Overall, all of the climatic factors show a yearly cyclical pattern in average monthly temperature, relative humidity, and rainfall, as shown in Figure $4 b-d$, respectively. Figure $4 a$ shows that dengue cases also follow a seasonal pattern, with incidences being highest during the monsoon season and lowest during the pre-monsoon season (January-April) in alternating years.

The autocorrelation and partial autocorrelation plots of dengue cases, monthly average temperature, monthly average relative humidity and monthly average rainfall for the 13-year period are shown in Figure S3. ACF gives us values of autocorrelation of any series (such as, temperature) with its lagged values. As depicted in Figure S3 there is a significant correlation at lags 1 and 2 followed by correlations that are not significant. This pattern indicates a moving average (MA) process of order 2. Other Figures clearly indicate the seasonal behavior of the monthly temperature, relative humidity and rainfall.

Second, when we checked the ACF and PACF plots of the residuals for the 13-year period to determine seasonal variations in the climatic parameters (Figure S4), we found an autoregressive pattern of order 1 . To account for this, we fitted the Poisson regression model (3) using the R-function tsglm from the tscount R-package.

From the fitted model output, the estimated coefficient and its standard error for temperature were found to be 0.10 and 0.003 , respectively. The confidence interval for the coefficient of temperature is $(0.10,0.11)$ which did not include zero. This means that the temperature is significant at a $5 \%$ level; that is, temperature plays a significant role in the number of dengue cases in the city of Dhaka. Since the estimate is positive, the 
number of dengue cases increases as the temperature increases, while adjusting for relative humidity and rainfall. For relative humidity, the estimate is 0.23 and the standard error is 0.002. The confidence interval for the coefficient of relative humidity is $(0.22,0.24)$ which did not include zero. This means that relative humidity is also significant at the $5 \%$ level and plays a significant role in the number of dengue cases in the city of Dhaka. As the estimate is positive, the number of dengue cases increases as relative humidity increases, while adjusting for temperature and rainfall. For rainfall, the estimate is -0.10 and the standard error is 0.002 . The confidence interval for the coefficient of rainfall is $(-0.10$, -0.09 ) which does not include zero. This means that rainfall is significant at the $5 \%$ level; that is, rainfall plays a significant role in the number of dengue cases in the city of Dhaka. As the estimate was negative, the dengue cases decrease as the rainfall increases, while adjusting for temperature and relative humidity.

\subsection{Analysis of Climate Anomaly vs. Dengue Cases}

After the seasonality analysis for dengue cases, we aspired to test the effects of climate anomalies on dengue cases. For this, we calculated the temperature anomaly using the relation (4) and plotted it in Figure 5 against dengue cases for the period of 2001-2013.

\section{Temperature anomaly vs Dengue cases}

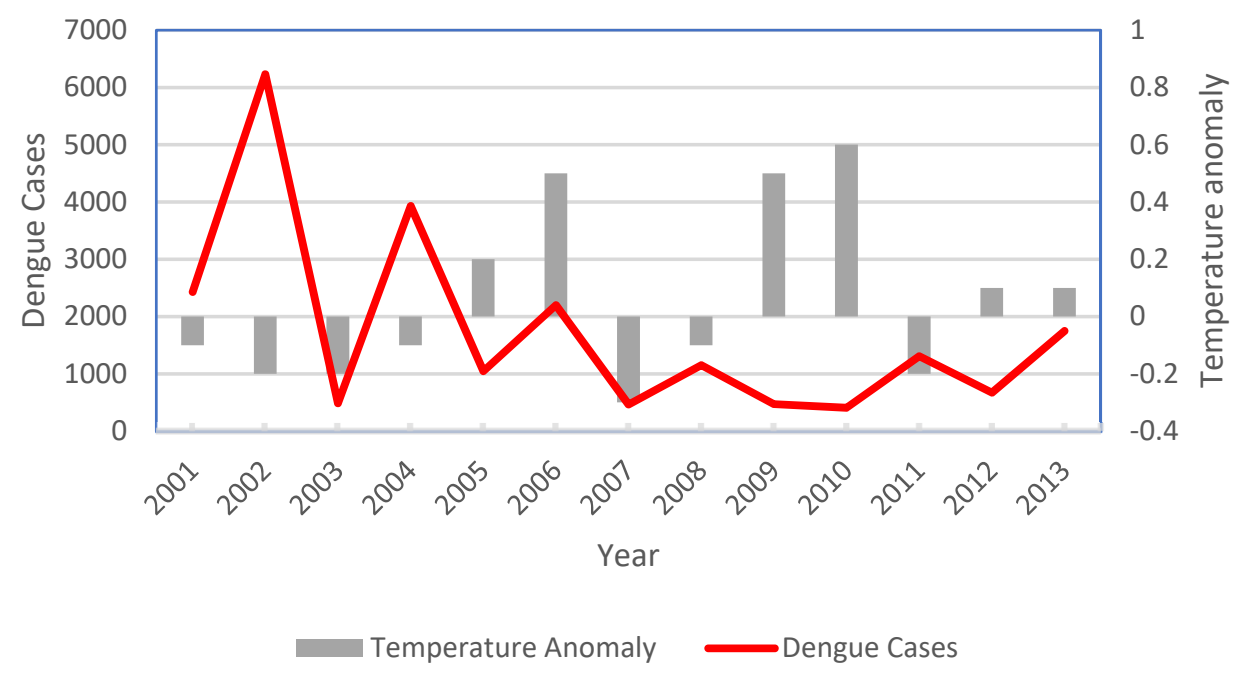

Figure 5. Distribution of temperature anomaly vs. dengue cases in the city of Dhaka, Bangladesh for the 13-year study period (2001-2013).

As shown in Figure 5, when the temperature anomaly was $-0.1^{\circ} \mathrm{C}$ (average yearly temperature $25.9^{\circ} \mathrm{C}$ for years 2001,2004 and 2008), or the anomaly was $-0.2^{\circ} \mathrm{C}$ (average yearly temperature $25.8^{\circ} \mathrm{C}$ in 2002, 2003 and 2011), the number of dengue cases was higher than the number of dengue cases when the climate average temperature $\left(26{ }^{\circ} \mathrm{C}\right)$ (1985-2013) occurs.

We also observed that at higher anomalies of $0.1-0.6^{\circ} \mathrm{C}$ (temperatures rise to $26.1-26.6^{\circ} \mathrm{C}$ in years 2005, 2006, 2009, 2010, 2012 and 2013), the dengue cases were lower than the dengue cases in an average climate temperature $\left(26^{\circ} \mathrm{C}\right)$. Thus, the number of dengue cases in the city of Dhaka declined when the average yearly temperature was $26^{\circ} \mathrm{C}$ and higher.

We calculated the rainfall anomaly using the relation (4) and plotted it against dengue cases in Figure 6 for the 2001-2013 period. The dengue cases tended to be higher for the years with a rainfall anomaly of -38.18 (annual average rainfall of $133 \mathrm{~mm}$ in 2013) and -10.76 anomaly (160 mm rainfall in 2006) as compared with the number of dengue cases for the average rainfall of $170 \mathrm{~mm}$ during the 29-year period of 1985-2013. 


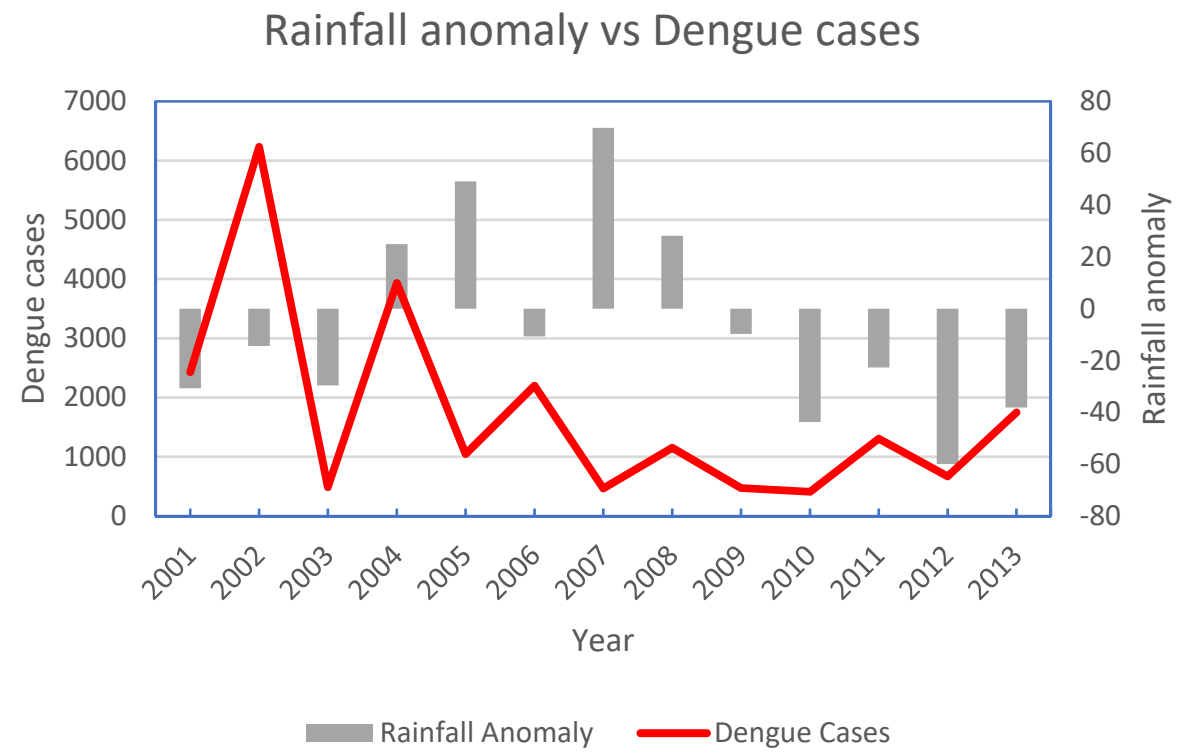

Figure 6. Distribution of rainfall anomaly vs. dengue cases in the city of Dhaka, Bangladesh for the 13-year study period (2001-2013).

We also observed that at higher rainfall anomalies, such as +69.74 in 2007 (rainfall of $240 \mathrm{~mm}$ ), the dengue cases tended to be lower than the number of dengue cases for the average rainfall. Based on the data collected, we infer that with an increase in the amount of rainfall (more than annual average of $200 \mathrm{~mm}$ ), the number of dengue cases tend to decline.

\section{Discussion}

Departing from conventional bivariate analytical approaches, in this study we undertook a step-by-step approach to determine the relationships between climatic factors (i.e., temperature, relative humidity and rainfall) and Stegomyia indices $[9,10,48,49]$, and subsequently between Stegomyia indices and dengue cases in the city of Dhaka, Bangladesh. Besides analyzing the effects of seasonal variability and climate anomalies on dengue cases, our study sought to develop a more complete understanding of the effects of climate on dengue cases by viewing the climate-vector-disease nexus as a sequential, step-bystep process.

In designing our study, we drew from Githeko's [50] study in Bangladesh. Githeko [50] observed that most previous studies on dengue only considered climatic or biological effects in isolation and emphasized that since climatic factors directly influence the breeding and prevalence of dengue vector mosquitos (e.g., Ae. aegypti), it was vital to study the effect of climate on the biological domain in order to fully understand the dynamics of dengue outbreaks. Following this recommendation, we attempted to determine the relationships within the climate-vector-dengue nexus, with the aim of meeting the objectives of this study.

In regard to the first objective, the results of our study provide evidence that overall dengue vector abundance is significantly influenced by climatic factors, though the significance of this relationship varies for each index and pair of factors. We found that mean rainfall (MR) and lag mean rainfall (LMR) at one month were positively and significantly related to Container Index (CI), i.e., an overwhelming majority (i.e., 79\%) of the variability in CI could be explained by MR and LMR. The mean rainfall contributes to the development of the Ae. aegypti when the daily or weekly mean rainfall is adequate for creating the breeding site as well as retaining the water for the growth of Ae. aegypti larvae and pupae. The lag mean rainfall indicates how much time needs to be passed after the actual rainfall to create the breeding site and having actual larval and pupal growth in the site. The mean 
relative humidity $(\mathrm{MH})$ and mean rainfall $(\mathrm{MR})$ were also found to be significantly related to the Breteau Index (BI), although MH was negatively associated with BI.

The regression model reveals that climatic factors account for about $72 \%$ of the variability in BI, which aligns with conclusions drawn by several other studies conducted in tropical regions $[8,9,13,48,51]$. However, while previous studies made the link between rainfall, water levels in indoor and outdoor containers, and the consequent increase in vector breeding and density and dengue prevalence, our findings and that of several other studies revealed that the dynamics of dengue spread are not so straightforward, and that the effects of temperature and relative humidity were also significant and complex.

Several comparable studies in Vietnam, Ecuador, Taiwan, Thailand, and China, which investigated the relationships between Stegomyia indices and Aedes abundance, provide useful insight into the impact of weather conditions on mosquito ecology. Pham et al.'s [8] study in the central high province of Vietnam confirmed a positive association between $\mathrm{HI}, \mathrm{BI}$, and $\mathrm{CI}$ and elevated temperatures, high humidity and rainfall, and a negative association with hours of sunlight [8]. Stewart-Ibarra et al. [10], in an empirical investigation of Ae. aegypti in Ecuador, confirmed that mosquito oviposition (egg-laying) was significantly driven by rainfall and minimum temperature. Tseng et al. [9] in their study of Taiwan found that temperature, lagged rainfall, and lagged density levels had positive and significant effects on the density of the mosquito population. Similar to these studies, the results of the present study in the city of Dhaka, Bangladesh revealed positive and significant relation of Stegomyia indices and the Aedes abundance.

Both Nakhapakorn and Tripathi [52] and Naish et al. [13] found that in Thailand, humidity is a crucial variable in the spread of dengue as high humidity—along with high temperatures and the presence of stagnant water-creates ideal breeding conditions for Ae. aegypti. Cheng et al. [51] examined the climate-vector abundance relationship in terms of the risk of dengue outbreak in Guangzhou, China, and found that that high precipitation during the monsoon increases vector abundance, but early and more frequent intervention and less vertical transmission can reduce the risk of dengue outbreak in successive time periods. Our study also corroborates these inferences by showing significant relationship between Stegomyia indices and the climate variables.

The second objective of our study was to determine the relationship between DF/SDF cases and vector abundance, as established by the parameters of the Stegomyia indicesa common epidemiological research framework that has been widely used in tropical areas $[9,10,48,49]$. Our empirical investigation in the city of Dhaka, Bangladesh has revealed that dengue cases in the city are significantly related to Stegomyia indices. We also found that $\mathrm{CI}$ is the most significant index, associated with dengue incidence, accounting for $49 \%$ of the variability in dengue cases.

In the context of Dhaka, these findings underscore the pivotal role of rainfall and stagnant water, relative to temperature and relative humidity, in affecting vector abundance and dengue incidence, as $\mathrm{CI}$ reflects the percentage of water-holding containers infected with larvae and/or pupae. These results are supported by Stewart-Ibarra et al.'s [10] study in Ecuador, that found mosquito oviposition was significantly driven by rainfall and minimum temperature, and that areas with large numbers of water storage containers played a major role for pupal development - the single most significant predictor of dengue outbreaks.

Previous studies on vector-dengue incidence have put forward two main philosophies regarding disease prevention and management. One school of thought argues that since dengue incidence is mainly driven by higher vector breeding associated with high temperature and rainfall, the policy focus should be on vector control. Pham et al. [8], for example, observed that in Vietnam the incidence of dengue fever is significantly and positively associated with Stegomyia indices. They argued that because higher vector abundance leads to higher rates of dengue infection, it is of utmost importance to control mosquitos during periods of high temperature and rainfall to reduce the risk of any outbreak. Similarly, the Barrera, Amador and MacKay's [48] study in San Juan, Puerto Rico, asserted that although 
both weather and anthropogenic activities are responsible for the abundance of Ae. aegypti, it is oviposition which is significantly correlated with dengue incidence, and which requires immediate attention.

Another school of thought asserts that Stegomyia indices, especially larval indices, are not sufficient to explain the vector-disease incidence relationship. Bowman et al. [49] reviewed the evidence systematically and concluded that the association between Stegomyia indices and dengue transmission was insufficient to predict an outbreak and called for the use of standardized study design and routine adult mosquito sampling in order to better understand vector ecology. In their study of the city of Kaohsiung, Taiwan, Chang et al. [53] came to a similar conclusion and argued that there may be different outbreak thresholds for different regions, local ecologies and herd immunity levels. As our findings revealed the relationship between vector abundance and dengue cases to account for only $49 \%$ of the variability in dengue incidence in the city of Dhaka, this study leaves room for other factors such as differences in adult mosquito numbers, infection rate of the adult mosquito, extrinsic incubation period, herd immunity, and population density, to be integrated into future epidemiological models for dengue.

The third objective of our study was to determine the relationship between climatic factors and DF/SDF cases. As the majority of studies on the climatic dimensions of dengue emergence ignored the entomological dimension, we pursued a longitudinal study of the city of Dhaka in order to more fully understand the disease propagation complex. The results of our generalized linear model reveal that, when the relationships between dengue cases and climatic factors during monsoon months over a 12-year period are tested, mean relative humidity and lag mean rainfall (i.e., rainfall at one-month lag) were found to be significant. The model explains $93 \%$ of the variability in dengue cases while considering climatic factors. As noted earlier, Karim et al.'s [32] study in Dhaka confirmed that rainfall, maximum temperature and relative humidity can explain $61 \%$ of the variability in reported dengue cases at a two-month lag. The study also found that the monsoon season, with a peak in August, was highly predictive of most reported dengue cases in Dhaka.

It is evident from both Karim et al.'s [32] and our findings that out of all climatic factors, rainfall has the most influence on dengue incidence in Dhaka. Arcari et al. [54] in Indonesia and Johansson et al. [55] in Puerto Rico also observed a strong positive relationship between rainfall and dengue incidence. Nonetheless, several investigations in Asia and Latin America concluded that the relationship between precipitation and dengue incidence may not be linear, as excess rainfall can negatively impact vector breeding $[14,15]$. Similar effects were predicted in Dhaka, where continuous heavy rainfall has the potential to flood Aedes breeding sites and wash larvae out into fast-flowing rivers, killing them. A significant negative observed association between rainfall and dengue cases, which is reflected in the confidence interval for the co-efficient of rainfall, confirms-albeit indirectly - the negative effects of rainfall on vector abundance and dengue cases. The confidence interval for the coefficients of temperature and relative humidity were positively and significantly associated with dengue cases in Dhaka.

Both short $(<5$ weeks) and long lag times between climatic factors and increasing dengue incidence have previously been confirmed by studies in Bangladesh $[31,32,56]$ and in Sri Lanka. In Dhaka, Hashizume et al. [31] explain that dengue incidence rarely rises immediately following heavy rainfall. The lag time between rainfall and emerging dengue cases, however, can vary due to several factors. The typical short ( $<5$ week) lag results directly from the life cycle of Aedes mosquitos. Heavy rainfall may leave stagnant pools of water on the ground or in objects such as discarded tires, which are ideal habitats for mosquito breeding. The lag time of one month that we found in the present study reflects a relatively more straightforward model of causation-water is required for breeding. Longer models, however, require a different explanation, that is, longer lag times indicate that weather can occur in cycles, and these longer lag times are an artefact solely of the relationship between weather events, rather than between weather events and the thriving of vectors. 
Deviations from this standard lag time, however, can emerge from variation in geographic location, elevation, humidity, temperature, infection rate of mosquitoes and other environmental factors. For instance, while in Bangladesh we mostly observed a short lag time ( $<5$ weeks), in our case it was one month, in Sri Lanka, Ehelepola et al. [57] observed an average 5-7-week lag, positively correlated with rainfall, temperature, humidity and hours of sunshine (but negatively correlated with wind). Based on an investigation of dengue incidence in Hanoi, Vietnam, Do et al. [58] distinguished between lag times caused by temperature and rainfall ( $8-10$ weeks lag) and by relative humidity (18 weeks).

The final objective of our study was to determine the pattern of seasonality and climate anomalies and their effects on DF/SDF cases. To this end, we undertook a longitudinal study covering a 13-year period (2001-2013) and assessing temperature, rainfall and relative humidity. Considering significant relationships of dengue incidence with climatic factors, longitudinal studies in terms of seasonality in dengue cases and their variation interannually has only recently received wider attention $[9,59,60]$. In our study, dengue cases exhibited a yearly cyclical pattern, with higher incidence beginning in the monsoon season (June-October), reaching a peak in August and minimum in the pre-monsoon period of January-April in alternate years.

The findings of our longitudinal study revealed that in Dhaka, temperature has a profound effect on vector breeding and DENV spread. The plot of calculated temperature anomalies vs. dengue cases for 2001-2013 exhibits that when the average yearly temperature was $25.9^{\circ} \mathrm{C}\left(-0.1^{\circ} \mathrm{C}\right.$ anomaly $)$ or $25.8^{\circ} \mathrm{C}\left(-0.2{ }^{\circ} \mathrm{C}\right.$ anomaly $)$, dengue cases were higher than the average. Contrary to our findings, Beebe et al.'s [61] study in Australia offers an explanation for such effects: higher temperatures in summer months force residents to store water in open containers in their homes, providing ideal breeding sites for Aedes mosquitos.

A number of studies worldwide showed strong direct association between temperature and Aedes abundance. For example, a study in Brazil revealed that the seasonal pattern of abundance of the Aedes mosquito is visible while having the temperature as a parameter at the city level [62]. In Thailand, Chavez et al. [63] observed that Aedes mosquito abundance changes considerably with temperature change. Contrary to these findings, an Australian study showed that the development of the immature Aedes mosquito was inversely associated with temperature [64]. Conforming with these, the present study in the city of Dhaka, Bangladesh, the mosquito abundance and dengue incidence were observed to be strongly associated with temperature changes. In Dhaka, when annual average temperatures reached $26.1-26.6^{\circ} \mathrm{C}$, dengue incidence was lower than the dengue incidence during "normal" temperatures $\left(26^{\circ} \mathrm{C}\right)$. Overall, our study revealed that dengue incidence rises with annual average temperature up to $26^{\circ} \mathrm{C}$, after which it begins to drop off.

In a study conducted in Bangladesh, Banu et al. [65] found a highly significant association between local climate variables (temperature and rainfall) and dengue incidence. However, when they studied the association between ENSO, IOD and dengue incidence, they observed that the extent of the association was very weak. They also reported that the association between dengue incidence and ENSO or IOD were comparatively stronger after an adjustment for local climate variables, seasonality and trend, when they applied a distributed lag nonlinear model (DLNM). Thus, Banu et al. [64] revealed that stronger effects of local climate variables propel the weak association between ENSO, IOD and dengue incidence, making the local climate variables more important for dengue incidence in Bangladesh.

Similar studies conducted in Puerto Rico, Thailand and Mexico by Johansson et al. [66] also observed a weak association between dengue and ENSO. In an earlier study, Hales et al. [67] found in the 14 island nations of the Pacific that dengue incidence was positively associated with ENSO in 10 of these island countries. Other studies in Thailand and Mexico also reported a positive association between dengue and ENSO [68,69]. Though the study of association between ENSO, IOD and dengue incidence were beyond the scope our study, it 
is worth noting that the effects of local climatic variables, weather conditions, and other factors regulating dengue dynamics indicate conformity with our study which revealed a strong association of the main climatic variables (temperature, rainfall and humidity) with dengue cases in the city of Dhaka, Bangladesh.

There are some limitations to this study. First, we were constrained by missing data as continuous time series data on dengue cases for the study period were not available in the government depository on dengue surveillance. To overcome this limitation and attain the best possible 'close approximation', the missing data were denoted as "missing at random". Following Little and Rubin [41] and Weerasinghe [42], these missing data were imputed by applying the Spline Interpolation Method (see R-package "imputTS") [43,44] and the regression imputation method [45]. Thus, the data limitation would not pose any serious constraint to the generalizability and future study directions. Second, the entomological surveys that collected data on Aedes larvae from households in Dhaka did not cover the same areas throughout the study period. We therefore relied on adjusted data for specific areas which were covered repeatedly by all survey periods over a 5-year period. Third, the entomological data did not cover adult Aedes mosquitoes, so, we could not estimate the extrinsic incubation period and the infection rate. Fourth, due to the unavailability of time-series data on other variables, we were unable to incorporate other contributing factors such as household water-use, vector control measures, and land-use changes into our models. Further empirical research on longitudinal trends in seasonality and its effects upon dengue vector breeding will assist with improved understanding of dengue disease transformation.

\section{Conclusions}

Recognizing that only nominal attempts have thus far been made to empirically examine the dynamic causal relationships within the climate-vector-disease nexus, the present study applied an innovative step-by-step approach to determine the causal relationships between climatic factors, dengue vector abundance, and dengue cases/incidence in the city of Dhaka, Bangladesh. Some of our results are similar to those of other studies, confirming that there is a significant correlation between climatic factors and vector abundance $[9,13,48,51]$, and between vector abundance and dengue incidence $[9,10,50]$. The examination of climate-vector-disease nexus of our study under one conceptual framework is a unique attempt and thus makes a novel contribution to this research domain concerning dengue disease transmission.

The integration of climate and entomological data into climate-vector-disease nexus to produce predictive models has not yet been fully realized [31]. In this regard, the present study provides evidence of a strong relationship amongst climatic factors, vector and dengue via a step-by-step process-revealing their significance in dengue vector abundance and dengue disease occurrence.

We found that an increase in Aedes abundance is associated with the rise in temperature, relative humidity, and rainfall during the monsoon months that in turn appear in the subsequent increase in dengue incidence. The relationships of dengue cases with Stegomyia indices as well as with the mean relative humidity and the lag mean rainfall were also highly significant. This study thus lays a strong foundation for future work on dengue forecasting and prevention, which may prove vital as the climate continues to change and the range and seasonal dynamics of dengue and other diseases change with it. As Wilder-Smith et al. [70] highlighted, the predictive models of the relationships between climate variables and dengue transmission that we pursued in the present study can assist in developing early warning systems. Based on the existing literature, we primarily focused on associations between climate data and Aedes abundance for the monsoon months. Future study should expand such entomological study for the non-monsoon months as well.

Supplementary Materials: The following are available online at https: / www.mdpi.com/article/10 .3390/atmos12070905/s1, Figure S1: ACF and PACF plots for CI in relation to the climatic parameters. The PACF plot indicating that the residuals in the fitted model were uncorrelated with mean zero 
and constant variance; Figure S2: ACF and PACF plots for BI in relation to the climatic parameters. The PACF plot indicating that the residuals in the fitted model were uncorrelated with mean zero and constant variance; Figure S3. Autocorrelation and partial autocorrelation plots for the number of dengue cases vs. climate factors by month for the 13-year study period (2001-2013): (a and b) number of dengue cases: (c and d) average temperature; (e and f) average relative humidity; ( $g$ and h) average rainfall; Figure S4. Autocorrelation and partial autocorrelation plot of residuals in the city of Dhaka, Bangladesh for the 13-year study period (2001-2013).

Author Contributions: Conceptualization, S.I. and C.E.H.; methodology, S.I., C.E.H. and S.H.; software, S.H. and S.I.; validation, S.I., S.H. and C.E.H.; formal analysis, S.I., S.H., C.E.H. and J.H.; investigation, S.I. and C.E.H.; resources, S.I., C.E.H., S.H. and J.H.; data curation, S.H. and S.I.; writing-original draft preparation, S.I., C.E.H. and S.H.; writing-review and editing, S.I., C.E.H., S.H. and J.H.; visualization, S.I., C.E.H.; supervision, C.E.H. and S.H.; project administration, C.E.H.; funding acquisition, S.I. and C.E.H. All authors have read and agreed to the published version of the manuscript.

Funding: This research was made possible through funding from the International Development Research Centre (IDRC), Ottawa, Canada (Grant \# 106040-001 to the second author); University of Manitoba, Winnipeg, Canada; North South University, Dhaka, and Bangladesh, International Center for Diarrheal Disease Research, Bangladesh (icddr,b), Dhaka, Bangladesh. Further financial assistance was received from the Social Science and Humanities Research Council, InSight Grant (Grant \# 435-2012-1748), Ottawa, Canada; and from International Development Research Centre (IDRC), Ottawa, Canada, through the IDRC Doctoral Research Awards (IDRA) (107099-050) to the first author.

Institutional Review Board Statement: This research was approved by the Bangladesh Medical Research Council (Bangladesh) and the Joint Faculty Research Ethics Board of the University of Manitoba (Canada). Administrative permission was granted by the Government of Bangladesh to access and use the meteorological data from the Bangladesh Meteorological Department, and entomological and dengue case data from the Directorate of Public Health and Services.

Informed Consent Statement: Not applicable.

Data Availability Statement: The datasets used and/or analyzed during the current study are available from the corresponding author on reasonable request.

Acknowledgments: This research was funded by the International Development Research Centre (IDRC), Canada (grant \# 106040-01 to the 2nd author) and (grant \# 107099-050 to the 1st author); and the Social Sciences and Humanities Research Council (SSHRC) of Canada (grant \# 435-2018-552) to the 2nd author; we thank these organizations for their financial supports. We are grateful to the Study Participants in Dhaka city communities for having confidence and trust in the interviewers and for sharing personal and household information with them. We are also appreciative of the Bangladesh Meteorological Department and Directorate General of Public Health and Services, Government of Bangladesh for providing us the required data and access to other necessary information and Gilles Messier for his assistance in editing the text.

Conflicts of Interest: The authors declare that they have no competing interest. The funders had no role in study design, data collection and analysis, decision to publish, or preparation of the manuscript.

\section{References}

1. Halstead, S.B. Dengue. Lancet 2007, 370, 1644-1652. [CrossRef]

2. Gubler, D.J. The global pandemic of dengue/dengue haemorrhagic fever: Current status and prospects for the future. Ann. Acad. Med. Singap. 1998, 27, 227-234. [PubMed]

3. Rodhain, F. Les insectes ne connaissent pas nos frontières. Médecine Mal. Infect. 1996, 26 (Suppl. 3), 408-414. [CrossRef]

4. WHO (World Health Organization). Dengue and Severe Dengue. 2021. Available online: https://www.who.int/news-room/ fact-sheets/detail/dengue-and-severe-dengue (accessed on 6 July 2021).

5. Githeko, A.K.; Lindsay, S.W.; Confalonieri, U.E.; Patz, J.A. Climate change and vector-borne diseases: A regional analysis. Bull. World Health Organ. 2000, 78, 1136.

6. Lorenz, C.; Azevedo, T.S.; Virginio, F.; Aguiar, B.S.; Chiaravalloti-Neto, F.; Suesdek, L. Impact of environmental factors on neglected emerging arboviral diseases. PLoS Negl. Trop. Dis. 2017, 11, e0005959. [CrossRef] 
7. Mcmichael, A.J.; Woodruff, R.E.; Hales, S. Climate change and human health: Present and future risks. Lancet 2006, 367, 859-869. [CrossRef]

8. Pham, H.V.; Doan, H.T.M.; Phan, T.T.T.; Minh, N.N.T. Ecological factors associated with dengue fever in a central highlands Province, Vietnam. BMC Infect. Dis. 2011, 11, 172. [CrossRef]

9. Tseng, W.-C.; Chen, C.-C.; Chang, C.-C.; Chu, Y.-H. Estimating the economic impacts of climate change on infectious diseases: A case study on dengue fever in Taiwan. Clim. Chang. 2009, 92, 123-140. [CrossRef]

10. Stewart Ibarra, A.M.; Ryan, S.J.; Beltrán, E.; Mejía, R.; Silva, M.; Muñoz, Á. Dengue vector dynamics (Aedes aegypti) influenced by climate and social factors in Ecuador: Implications for targeted control. PLoS ONE 2013, 8, e78263. [CrossRef]

11. Depradine, C.A.; Lovell, E.H. Climatological variables and the incidence of dengue disease in Barbados. Int. J. Environ. Health Res. 2004, 14, 429-441. [CrossRef]

12. Colón-González, F.J.; Fezzi, C.; Lake, I.R.; Hunter, P.R. The effects of weather and climate change on dengue. PLoS Negl. Trop. Dis. 2013, 7, e2503. [CrossRef]

13. Naish, S.; Dale, P.; Mackenzie, J.S.; McBride, J.; Mengersen, K.; Tong, S. Climate change and dengue: A critical and systematic review of quantitative modelling approaches. BMC Infect. Dis. 2014, 14, 167. [CrossRef]

14. Halide, H.; Ridd, P. A predictive model for dengue hemorrhagic fever epidemics. Int. J. Environ. Health Res. 2008, 18, 253-265. [CrossRef]

15. Wu, P.-C.; Lay, J.-G.; Guo, H.-R.; Lin, C.-Y.; Lung, S.-C.; Su, H.-J. Higher temperature and urbanization affect the spatial patterns of dengue fever transmission in subtropical Taiwan. Sci. Total Environ. 2009, 407, 2224-2233. [CrossRef] [PubMed]

16. Islam, S.; Haque, C.E.; Hossain, S.; Rochon, K. Role of container type, behavioural, and ecological factors in Aedes pupal production in Dhaka, Bangladesh: An application of zero-inflated negative binomial model. Acta Trop. 2019, 193, 50-59. [CrossRef] [PubMed]

17. Kuno, G. Review of the factors modulating dengue transmission. Epidemiol. Rev. 1995, 17, 321-335. [CrossRef]

18. Wu, J.-Y.; Lun, Z.-R.; James, A.A.; Chen, X.-G. Review: Dengue fever in mainland China. Am. J. Trop. Med. Hyg. 2010, 83, 664-671. [CrossRef] [PubMed]

19. Yu, H.-L.; Yang, S.-J.; Yen, H.-J.; Christakos, G. A spatio-temporal climate-based model of early dengue fever warning in southern Taiwan. Stoch Environ. Res. Risk Assess 2011, 25, 485-494. [CrossRef]

20. Shahid, S.; Harun, S.B.; Katimon, A. Changes in diurnal temperature range in Bangladesh during the time period 1961-2008. Atmos. Res. 2012, 118, 260-270. [CrossRef]

21. Yunus, E.B. Dengue outbreak 2000: The emerged issues. Bangladesh Med. J. 2000, 33, 46-47.

22. Hossain, M.A.; Khatun, M.; Arjumand, F.; Nisalak, A.; Breiman, R.F. Serologic evidence of dengue infection before onset of epidemic, Bangladesh. Emerg. Infect. Dis. 2003, 9, 1411-1414. [CrossRef]

23. Yunus, E.B.; Bangali, A.M.; Mahmood, M.A.H.; Rahman, M.M.; Chowdhury, A.R.; Talukder, K.R. Dengue outbreak 2000 in Bangladesh: From speculation to reality and exercises. Dengue Bull. 2001, 25, 15-20.

24. Sharmin, S.; Glass, K.; Viennet, E.; Harley, D. Interaction of mean temperature and daily fluctuation influences dengue incidence in Dhaka, Bangladesh. PLoS Negl. Trop. Dis. 2015, 9, e0003901. [CrossRef]

25. Rahman, M.; Rahman, K.; Siddque, A.K.; Shoma, S.; Kamal, A.H.; Ali, K.S.; Nisaluk, A.; Breiman, R.F. First outbreak of dengue hemorrhagic fever, Bangladesh. Emerg. Infect. Dis. 2002, 8, 738-740. [CrossRef] [PubMed]

26. Morales, I.; Salje, H.; Saha, S.; Gurley, E.S. Seasonal distribution and climatic correlates of dengue disease in Dhaka, Bangladesh. Am. J. Trop. Med. Hyg. 2016, 94, 1359-1361. [CrossRef] [PubMed]

27. Dhaka Tribune. DGHS: 673 More Hospitalized for Dengue in 24hrs. 13 September 2019. Available online: https://www. dhakatribune.com/bangladesh/2019/09/13/dghs-673-more-hospitalized-for-dengue-in-24hrs (accessed on 14 September 2019).

28. Shirin, T.; Muraduzzaman, A.K.; Alam, A.N.; Sultana, S.; Siddiqua, M.; Khan, M.H.; Akram, A.; Sharif, A.R.; Hossain, S.; Flora, M.S. Largest dengue outbreak of the decade with high fatality may be due to reemergence of DEN-3 serotype in Dhaka, Bangladesh, necessitating immediate public health attention. New Microbes New Infect. 2019, 29, 100511. [CrossRef]

29. Dhar-Chowdhury, P.; Paul, K.K.; Haque, C.E.; Hossain, S.; Lindsay, L.R.; Dibernardo, A.; Brooks, W.A.; Drebot, M.A. Dengue seroprevalence, seroconversion and risk factors in Dhaka, Bangladesh. PLoS Negl. Trop. Dis. 2017, 11, e0005475. [CrossRef]

30. Banu, S.; Hu, W.; Hurst, C.; Guo, Y.; Islam, M.Z.; Tong, S. Space-time clusters of dengue fever in Bangladesh. Trop. Med. Int. Health 2012, 17, 1086-1091. [CrossRef]

31. Hashizume, M.; Dewan, A.K.; Sunahara, T.; Rahman, M.Z.; Yamamoto, T. Hydroclimatological variability and dengue transmission in Dhaka, Bangladesh: A time-series study. BMC Infect. Dis. 2012, 12, 98. [CrossRef]

32. Karim, M.N.; Munshi, S.U.; Anwar, N.; Alam, M.S. Climatic factors influencing dengue cases in Dhaka city: A model for dengue prediction. Indian J. Med. Res. 2012, 136, 32-39.

33. Sharmin, S.; Viennet, E.; Glass, K.; Harley, D. The emergence of dengue in Bangladesh: Epidemiology, challenges and future disease risk. Trans. R. Soc. Trop. Med. Hyg. 2015, 109, 619-627. [CrossRef]

34. Paul, K.K.; Dhar-Chowdhury, P.; Haque, C.E.; Al-Amin, H.M.; Goswami, D.R.; Kafi, M.A.; Drebot, M.A.; Lindsay, L.R.; Ahsan, G.U.; Brooks, W.A. Risk factors for the presence of dengue vector mosquitoes, and determinants of their prevalence and larval site selection in Dhaka, Bangladesh. PLoS ONE 2018, 13, e0199457. [CrossRef] [PubMed]

35. Islam, M.Z.; Rutherford, S.; Dung Phung, M.; Uzzaman, N.; Baum, S.; Huda, M.M.; Asaduzzaman, M.; Talukder, M.R.; Chu, C. Correlates of climate variability and dengue fever in two metropolitan cities in Bangladesh. Cureus 2018, 10, e3398. [CrossRef] 
36. Ahmed, T.; Rahman, G.M.S.; Bashar, K.; Shamsuzzaman, M.; Samajpati, S.; Sultana, S.; Hossain, M.I.; Banu, N.N.; Rahman, M.S. Seasonal prevalence of dengue vector mosquitoes in Dhaka City, Bangladesh. Bangladesh J. Zool. 2007, 35, $205-212$.

37. BBS (Bangladesh Bureau of Statistics). Statistical Yearbook 2016; BBS, Ministry of Planning: Dhaka, Bangladesh, 2016.

38. UN (United Nations). The World's Cities in 2016: Data Booklet. 2016. Available online: http://www.un.org/en/development/ desa/population/publications/pdf/urbanization/the_worlds_cities_in_2016_data_booklet.pdf (accessed on 3 June 2020).

39. BBS (Bangladesh Bureau of Statistics). Statistical Yearbook 2010; BBS, Ministry of Planning: Dhaka, Bangladesh, 2010.

40. WMO (World Meteorological Organization). WMO Guidelines on the Calculation of Climate Normals, 2017 ed.; WMO-No. 1203; WMO: Geneva, Switzerland, 2017; p. 29.

41. Little, R.J.A.; Rubin, D.B. Statistical Analysis with Missing Data, 2nd ed.; John Wiley: New York, NY, USA, 2002.

42. Weerasinghe, D.S.S. A missing values imputation method for time series data: An efficient method to investigate the health effects of sulphur dioxide levels. Environmetrics 2010, 21, 162-172. [CrossRef]

43. Wolberg, G. Cubic Spline Interpolation: A Review; Columbia University: New York, NY, USA, 1988.

44. Moritz SBartz-Beielstein, T. imputeTS: Time Series Missing Value Imputation in R. R J. 2017, 9, 207-218. [CrossRef]

45. Buuren, S.V. Flexible Imputation of Missing Data; Chapman and Hall/CRC: Boca Raton, FL, USA; New York, NY, USA, 2012.

46. R Core Team. R: A Language and Environment for Statistical Computing; R Foundation for Statistical Computing: Vienna, Austria, 2019; Available online: http:/ / www.R-project.org (accessed on 20 June 2020).

47. Wood, S.N. Generalized Additive Models: An Introduction with R; Chapman and Hall: Boca Raton, FL, USA, 2006.

48. Barrera, R.; Amador, M.; MacKay, A.J. Population dynamics of Aedes aegypti and dengue as Influenced by weather and human behavior in San Juan, Puerto Rico. PLoS Negl. Trop. Dis. 2011, 5, e1378. [CrossRef] [PubMed]

49. Bowman, L.R.; Runge-Ranzinger, S.; McCall, P.J. Assessing the relationship between vector indices and dengue transmission: A systematic review of the evidence. PLoS Negl. Trop. Dis. 2014, 8, e2848. [CrossRef]

50. Githeko, A.K. Advances in developing a climate-based dengue outbreak models in Dhaka, Bangladesh: Challenges and opportunities. Commentary. Indian J. Med. Res. 2012, 136, 7-9.

51. Cheng, Q.; Jing, Q.; Spear, R.C.; Marshall, J.M.; Yang, Z.; Gong, P. Climate and the timing of imported cases as determinants of the dengue outbreak in Guangzhou, 2014: Evidence from a mathematical model. PLoS Negl. Trop. Dis. 2016, 10, e0004417. [CrossRef]

52. Nakhapakorn, K.; Tripathi, N.K. An information value-based analysis of physical and climatic factors affecting dengue fever and dengue haemorrhagic fever incidence. Int. J. Health Geogr. 2005, 4, 13. [CrossRef] [PubMed]

53. Chang, F.-S.; Tseng, Y.-T.; Hsu, P.-S.; Chen, C.-D.; Lian, I.-B.; Chao, D.-Y. Re-assess vector indices threshold as an early warning tool for predicting dengue epidemic in a dengue non-endemic country. PLoS Negl. Trop. Dis. 2015, 9, e0004043. [CrossRef] [PubMed]

54. Arcari, P.; Tapper, N.; Pfueller, S. Regional variability in relationships between climate and dengue/SD in Indonesia. Singap. J. Trop. Geogr. 2007, 28, 251-272. [CrossRef]

55. Johansson, M.A.; Dominici, F.; Glass, G.E. Local and global effects of climate on dengue transmission in Puerto Rico. PLoS Negl. Trop. Dis. 2009, 3, e382. [CrossRef] [PubMed]

56. Muurlink, T.O.; Stephenson, P.; Islam, M.Z.; Taylor-Robinson, A.W. Long-term predictors of dengue outbreaks in Bangladesh: A data mining approach. Infect. Dis. Model. 2018, 3, 322-330. [CrossRef]

57. Ehelepola, N.D.B.; Ariyaratne, K.; Buddhadasa, W.M.N.P.; Ratnayake, S.; Wickramasinghe, M. A study of the correlation between dengue and weather in Kandy City, Sri Lanka (2003-2012) and lessons learned. Infect. Dis. Poverty 2015, 4, 42. [CrossRef]

58. Do, T.T.T.; Martens, P.; Luu, N.H.; Wright, P.; Choisy, M. Climatic-driven seasonality of emerging dengue fever in Hanoi, Vietnam. BMC Public Health 2014, 14, 1078. [CrossRef]

59. Hii, Y.L.; Zhu, H.; Ng, N.; Ng, L.C.; Rocklov, J. Forecast of dengue incidence using temperature and rainfall. PLoS Negl. Trop. Dis. 2012, 6, e1908. [CrossRef]

60. Bannister-Tyrrell, M.; Williams, C.; Ritchie, S.A.; Rau, G.; Lindesay, J.; Mercer, G.; Harley, D. Weather-driven variation in dengue activity in Australia examined using a process-based modeling approach. Am. J. Trop. Med. Hyg. 2013, 88, 65-72. [CrossRef]

61. Beebe, N.W.; Cooper, R.D.; Mottram, P.; Sweeney, A.W. Australia's dengue risk driven by human adaptation to climate change. PLoS Negl. Trop. Dis. 2009, 3, e429. [CrossRef]

62. Lana, R.M.; Morais, M.M.; Lima, T.F.; Carneiro, T.G.; Stolerman, L.M.; dos Santos, J.P.; Cortés, J.J.; Eiras, Á.E.; Codeço, C.T. Assessment of a trap based Aedes aegypti surveillance program using mathematical modeling. PLoS ONE 2018, 13 , e0190673. [CrossRef] [PubMed]

63. Chaves, L.F.; Morrison, A.C.; Kitron, U.D.; Scott, T.W. Nonlinear impacts of climatic variability on the density-dependent regulation of an insect vector of disease. Glob. Chang. Biol. 2012, 18, 457-468. [CrossRef]

64. Tun-Lin, W.; Burkot, T.R.; Kay, B.H. Effects of temperature and larval diet on development rates and survival of the dengue vector Aedes aegypti in north Queensland, Australia. Med. Vet. Entomol. 2000, 14, 31-37. [CrossRef]

65. Banu, S.; Guo, Y.; Hu, W.; Dale, P.; Mackenzie, J.S.; Mengersen, K.; Tong, S. Impacts of El Niño Southern Oscillation and Indian Ocean Dipole on dengue incidence in Bangladesh. Sci. Rep. 2015, 5, 16105. [CrossRef] [PubMed]

66. Johansson, M.A.; Cummings, D.A.T.; Glass, G.E. Multiyear climate variability and dengue-El Nino Southern Oscillation, weather, and dengue Incidence in Puerto Rico, Mexico, and Thailand: A longitudinal data analysis. PLoS Med. 2009, 6, e1000168. [CrossRef] 
67. Hales, S.; Weinstein, P.; Souares, Y.; Woodward, A. El Nino and the dynamics of vector-borne disease transmission. Environ. Health Perspect. 1999, 107, 99-102. [PubMed]

68. Tipayamongkholgul, M.; Fang, C.T.; Klinchan, S.; Liu, C.M.; King, C.C. Effects of the El Nino-Southern Oscillation on dengue epidemics in Thailand, 1996-2005. BMC Public Health 2009, 9, 422. [CrossRef]

69. Hurtado-Diaz, M.; Riojas-Rodriguez, H.; Gomez-Dantes, H.; Cifuentes, E. Short communication: Impact of climate variability on the incidence of dengue in Mexico. Trop. Med. Int. Health 2007, 12, 1327-1337. [CrossRef]

70. Wilder-Smith, A.; Renhorn, K.E.; Tissera, H.; Abu Bakar, S.; Alphey, L.; Kittayapong, P.; Lindsay, S.; Logan, J.; Hatz, C.; Reiter, P.; et al. Dengue tools: Innovative tools and strategies for the surveillance and control of dengue. Glob. Health Action 2012, $5,17273$. [CrossRef] 